\title{
The neuro-hematopoietic-inflammatory arterial axis: The missing link between PTSD and cardiovascular disease?
}

\author{
Aldo L. Schenone, $M D{ }^{a}{ }^{a}$ and Wael A. Jaber, $M D^{a}$ \\ a Heart and Vascular Institute, Department of Cardiovascular Medicine, Cleveland Clinic, \\ Cleveland, $\mathrm{OH}$
}

Received Apr 22, 2019; accepted Apr 23, 2019

doi: $10.1007 / \mathrm{s} 12350-019-01748-2$

\section{See related article, pp. 688-694}

Psychological trauma with its associated post-traumatic stress disorder (PTSD) is a debilitating disorder which may lead to important psychiatric morbidity and devastating social consequences. Moreover, those living with PTSD are more likely to have chronic medical conditions such as obesity, smoking disorders, diabetes, and in particular cardiovascular disease (CVD). This observation has fueled recent research efforts to elucidate a mechanistic link between PTSD and CVD. ${ }^{1,2}$

Now that convincing evidence implicates inflammation as a key player in CVD, many have hypothesized that inflammation is the missing link between PTSD and cardiovascular events. ${ }^{3,4}$ This is supported by an emergent body of evidence indicating an immunological balance skewed toward a low-grade pro-inflammatory state in PTSD. ${ }^{1,5,6}$ Although findings have not been unanimous, the vast majority of studies has linked PTSD, with elevated level of pro-inflammatory markers (cytokines, acute-phase proteins, and/or white blood cells) and reduction in inhibitory cytokines. ${ }^{1,5}$ Nevertheless, this has been a source of ongoing debate given the lack of a standard definition of low-grade inflammation and notable methodological heterogeneity across studies. Furthermore, given the cross-sectional design of these studies, the high prevalence of comorbid conditions and established CVD, it is not possible to

Reprint requests: Wael A. Jaber, MD, Heart and Vascular Institute, Department of Cardiovascular Medicine, Cleveland Clinic, Cleveland, OH; jaberw@ccf.org

J Nucl Cardiol 2021;28:695-7.

$1071-3581 / \$ 34.00$

Copyright (c) 2019 American Society of Nuclear Cardiology. definitively establish causality and even less direction of causality.

It remains to be elucidated if inflammation precedes the onset of PTSD, identifying a vulnerable population, or rather ensues the onset of PTSD. ${ }^{5}$ Similarly, whether this inflammatory state is caused by an inappropriate response to perceived stress invoking dysregulation in the autonomic nervous system (ANS) and suppression of the hypothalamic-pituitary-adrenal (HPA) axis, or is produced by associated comorbid exposures and behaviors such as smoking, depression, obesity, physical exhaustion, and sleep deprivation among others remains unknown. ${ }^{1}$ Lastly, it is unclear how the time from index event, duration and severity of PTSD symptoms might affect a potential relationship to inflammation. ${ }^{5}$ Other confounders include heterogeneity and chronicity of events leading to PTSD (war, childhood trauma, sexual assault, etc.) as well as temporal proximity to precipitating events.

The pilot study by Toczek $\mathrm{et}^{\mathrm{al}}{ }^{7}$ in this issue of the Journal of Nuclear Cardiology offers relevant information to the ongoing debate regarding the interplay between PTSD, inflammation, and CVD. The authors investigated whether patients with PTSD exhibit enhanced systemic and vascular inflammation. The PTSD diagnosis and persistence of symptoms at time of imaging was confirmed using validated tools and questionnaires. ${ }^{18} \mathrm{~F}-\mathrm{FDG}$ PET/CT imaging was used to evaluate brain, hematopoietic, and arterial signals in subjects with or without PTSD. Sixteen 16 subjects (9 PTSD and 7 controls, age $34 \pm 7$ years) underwent ${ }^{18} \mathrm{~F}$ FDG PET/CT imaging with signals assessed in the ascending aorta (an index of arterial inflammation), amygdala (as a measure of stress-associated neurobiological activity), and spleen and bone marrow (hematopoietic activity). PTSD subjects were largely symptomatic (PCL-5 score $34 \pm 12$ ) at time of imaging with a mean time from index event of $16 \pm 13$ years. 
Neither circulating systemic inflammatory markers (TNF $\alpha$, IL-1 $\beta$, and IL-6) nor the aforementioned FDG signals differed between groups. From their findings, the authors concluded that their pilot study did not reveal any differential vascular or systemic inflammation as assessed by serum inflammatory markers and ${ }^{18} \mathrm{~F}-\mathrm{FDG}$ PET imaging between PTSD and healthy control subjects. Importantly, investigators observed significant positive correlations between (1) amygdala and hematopoietic (splenic and bone marrow) ${ }^{18}$ F-FDG signals, as well as, hematopoietic (splenic and bone marrow) and aorta signals. Nevertheless, no correlation of ${ }^{18} \mathrm{~F}$-FDG was found between amygdala and aorta.

Toczek et al are commended for enrolling a young cohort of patients with low prevalence of cardiac risk factor and no established CVD with attention to cardiovascular risk factors in an attempt to reduce confounders that have plagued previous epidemiological studies. ${ }^{1,2}$ Similarly, investigators are praised for documenting time from index event and documenting persistence of symptoms at time of imaging. We also applaud the adjunctive use of ${ }^{18} \mathrm{~F}$-FDG PET/CT along with the traditional serum inflammatory markers. This allowed the evaluation of arterial inflammation in vivo, a clinically relevant marker of unstable atherosclerotic plaque and predictor of future cardiovascular events. ${ }^{8,9}$ Moreover, the imaging of brain, hematopoietic, and arterial signals permitted ascertainment of the potential role of a neuro-hematopoietic-arterial axis in the PTSDinflammation-CVD trinity.

The negative results of this pilot study contrast with several far larger epidemiological studies that have previously shown that inflammation is increased among individuals with PTSD. It is quite possible that the lack of a difference between groups for any of the inflammatory variables may be the result of the small size of the cohort. Thus a more modest increase in systemic and arterial inflammation that was anticipated among PTSD subjects cannot be definitely excluded. In addition, the studied cohort likely represent a less-debilitated PTSD group of subjects, or possibly affected individuals on their way to recovery, as they volunteered to participate. This limits the extrapolation of the findings to the general PTSD population. Hence, it would take a large study with robust study design to debunk or confirm the observation of heightened inflammation among individuals with PTSD. Nevertheless, skeptics could argue that results may be, at least in part, explained by the enrollment of a younger population with a low prevalence of cardiovascular risk factors. If confirmed in a larger population, this findings may call into question the proposed pathophysiologic role of dysregulations in the ANS and HPA-axis and rather favor the theory of associated comorbid exposures and behaviors driving the low-grade pro-inflammatory state. Whether a more severe or longer duration of PTSD symptoms or PTSD treatment would have altered the results of this study or not remains uncertain. Another elegant but less realistic study design to address this relevant clinical question would be to include subjects before and after exposure to PTSD; troops before and after deployment to war zones and assess them prospectively to variables included in the study by Toczek et al.

The most interesting observation of this paper is that of a positive correlation between amygdalar and hematopoietic system activities, as well as, potential association between hematopoietic activity and arterial inflammation. This is notable since hematopoietic system activity has been linked to several downstream diseases including atherosclerosis as part of the so-called cardio-splenic axis. ${ }^{9}$ The unique finding here is that it is noted in a young cohort and so suggests that these systems may act over a large portion of a lifetime.

To conclude, the results of the Toczek et al innovative study $^{7}$ although not robust enough to change current paradigms on PTSD and inflammation, the findings are certainly intriguing and merit further investigation with larger studies.

\section{Disclosures}

The authors declare that they have no conflicts of interest.

\section{References}

1. Speer K, Upton D, Semple S, McKune A. Systemic low-grade inflammation in post-traumatic stress disorder: A systematic review. J Inflamm Res 2018. https://doi.org/10.3389/fphys.2018.01741.

2. Levine AB, Levine LM, Levine TB. Posttraumatic stress disorder and cardiometabolic disease. Cardiology 2013. https://doi.org/10. $1159 / 000354910$.

3. Ridker PM. The JUPITER trial: Results, controversies, and implications for prevention. Circ Cardiovasc Qual Outcomes 2009;2:279-85.

4. Ridker PM, Everett BM, Thuren T, MacFadyen JG, Chang WH, Ballantyne C, et al. Antiinflammatory therapy with canakinumab for atherosclerotic disease. N Engl J Med 2017;377:1119-31.

5. Wang Z, Caughron B, Young MRI. Posttraumatic stress disorder: An immunological disorder? Front Psychiatry 2017;8:222.

6. Mellon SH, Gautam A, Hammamieh R, Jett M, Wolkowitz OM. Metabolism, metabolomics, and inflammation in posttraumatic stress disorder. Biol Psychiatry 2018;83:866-75.

7. Toczek J, Hillmer A, Han J, Liu C, Peters D, Emami H, et al. FDG PET imaging of vascular inflammation in patients with posttraumatic stress disorder: A pilot case-control study. J Nucl Cardiol 2019;25:392-7.

8. Bucerius J, Dijkgraaf I, Mottaghy FM, Schurgers LJ. Target identification for the diagnosis and intervention of vulnerable atherosclerotic plaques beyond $18 \mathrm{~F}$-fluorodeoxyglucose positron emission tomography imaging: Promising tracers on the horizon. Eur J Nucl Med Mol Imaging 2019;46:251-65. 
9. Emami H, Singh P, Macnabb M, Vucic E, Lavender Z, Rudd JHF, et al. Splenic metabolic activity predicts risk of future cardiovascular events: Demonstration of a cardiosplenic axis in humans. JACC Cardiovasc Imaging 2015;8:121-30.
Publisher's Note Springer Nature remains neutral with regard to jurisdictional claims in published maps and institutional affiliations. 